\title{
Addressing Vaccination Hesitancy in Psychotherapy
}

\author{
Gary R. VandenBos
}

Published online: 1 February 2021

(C) National Register of Health Service Psychologists 2021

\begin{abstract}
Vaccine hesitancy refers to delay in acceptance or refusal of vaccination despite availability of vaccination series. The majority of psychologists reported that they do not anticipate resuming in-person service delivery until after a vaccine was available. There are currently no best practice guidelines for making the transition back to in-office practice after the COVID-19 pandemic. This article provides some initial thoughts on relevant dimensions to consider. These include utilization of the core CDC behavioral practices of mask use and social distancing and cleaning surfaces. Psychologists are also encouraged to set positive expectations about vaccination, state clear expectations of vaccination for in-office care, actively listen to patient doubts about vaccinations, and actively exploring patient understanding of the COVID-19 disease processes and prevention efforts.
\end{abstract}

\section{Vignette}

You have been practicing via telepsychology means for the last 11 months. You made a practice announcement a couple months ago that you would begin seeing vaccinated patients about a month after you yourself are vaccinated. It is time to begin discussing with your patients the option of returning to in-person, office-based care. You plan to continue to provide telepsychology sessions for unvaccinated patients. A few of your patients have expressed doubts (and fears) about the vaccine. A couple of patients have expressed extreme resistance to the idea of getting a vaccination, but also wish to return to in-office treatment. What do you do? Should you make an exception for them? Does that create ethical problems in terms of exposing other patients to increased risks? How might you best address these patient doubts and potential refusal to get vaccinated?

\section{COVID-19 Pandemic}

Three months after being initially detected in China, a new virus had migrated to the United States and was rapidly spreading. In March 2020, a nationwide closedown was encouraged as a public health measure. The professional practice of practice changed drastically. Almost overnight, $83 \%$ of psychologists reported using telepsychology almost exclusively - via Zoom, Doxy.me, and other platforms (Sammons et al., 2020a).
The pandemic continued, with the number of new cases exploding late in the year. By the end of 2020, 20 million Americans had tested positive for COVID19-with 346,000 deaths (Centers for Disease Control and Prevention, 2020a). Infections would continue into 2021.

Work developing a vaccine against the coronavirus was "fast-tracked" during 2020. On December 11, 2020, the US Food and Drug Administration issued the first emergency use authorization for a vaccine, developed by Pfizer-BioNTech, for the prevention of COVID-19. Seven days later a second vaccine, developed by Moderna, was approved for emergency use in the US. The next challenge will be vaccinating over 330 million Americans.

\section{Vaccine Hesitancy}

"Vaccine Hesitancy" refers to delay in acceptance or refusal of vaccination despite availability of vaccination series. Vaccine hesitancy is complex and context specific. It varies across time, setting, and vaccines (MacDonald and SAGE Working Group on Vaccine Hesitancy, 2015).

Human vaccination against preventable diseases is a relatively new process, only moving into major use in the last 70 years (Bloom et al., 2005). Strong progress was made on childhood vaccinations in the 1970s and 1980s. More recently, a small but growing group of parents have begun resisting such vaccinations (Lewis \& Speers, 2003). Addressing such beliefs is a common clinical challenge in pediatric medicine today (Leask, 2011). 


\section{Vaccine Hesitancy in the Context of COVID-19}

The worldwide scientific community went into high speed during 2020 to work on developing vaccines to prevent COVID-19. Ironically, one of the reasons cited by some individuals for being doubtful about the new vaccines is a concern that they are unsafe because they were developed so rapidly. It was the provision of a massive amount of funding that made rapid development possible. The vaccines have gone through all of the usual trials, steps, and reviews, and they have been found safe.

Nonetheless, many rumors about COVID-19 vaccines, and other vaccines, exist and spread without evidence and despite empirical demonstration that they are untrue (Larson, 2020). Some common misconceptions can be found on the Internet. A small sampling includes the following: severe side effects to the vaccine (untrue), changes to one's DNA (untrue, the two currently available vaccines work via mRNA), use of fetal tissue in development of vaccine (untrue), and that the vaccine will be used to introduce a microchip or nano transducer into the body (untrue).

\section{Returning to a "New Normal" After the Pandemic}

The availability of a vaccine offers the potential to return to some degree of a normal pattern of daily life for the average American. But to achieve this, the vast majority of the population will need to receive the vaccination. Thus, as 2021 begins, the US faces two simultaneous health challenges: to continue to fight against the spread of COVID-19 and to begin a massive vaccination program. With effective vaccinations, transition from pandemic conditions to the "new normal" should occur over the course of 9-12 months. These twin health initiatives will affect the practice of psychology.

The majority of psychologists reported that they do not anticipate resuming in-person service delivery until after a vaccine was available (Sammons et al., 2020b). There are currently (as of the beginning of 2021) no professional guidelines or best practices for making the transition back to inoffice practice. Each psychologist needs to determine for themselves when they will be ready to set the conditions under which they will or will not see a given patient. However, earlier CDC guidelines on COVID-19 prevention practices offer some help.

\section{CDC COVID-19 Prevention Guidelines}

Respiratory transmission is the key to COVID-19 transmission. The core elements of the CDC COVID-19 prevention guidelines all relate to a decrease in potential exposure to respiratory droplets containing the virus that causes COVID19 (Centers for Disease Control and Prevention, 2020b). Wearing a mask decreases the probability of an infected individual pushing such droplets out into the air around them (and to a lesser degree decreases the probability of an uninfected individual taking in such droplets). Keeping at least a six-foot social distance decreases the probability of each individual's breath carrying droplets to another individual. Avoiding crowds reduces the number of potentially infected individuals to which individuals are exposed. Droplets dissipate more readily in outdoor spaces, so poorly ventilated indoor spaces should be avoided as social gathering places.

Frequent handwashing (and hand sanitizer use) is also helpful. This removes droplets that might have been picked up by hands moving through contaminated air or droplets picked up from contaminated surfaces. The goal is to avoid introducing the virus into the body by touching the eyes, nose, or mouth with contaminated hands. Cleaning and disinfecting high-touch surfaces further helps to achieve such goals.

Thus, some initial expectations for in-person clinical care seem obvious. Both parties involved in psychotherapy should wear a mask. The chairs should be at least 6- 8 feet apart. One could consider placing a plexiglass partition between the patient and therapist. High-touch areas should be cleaned between sessions.

Individual therapy has a lower risk of exposure than group therapy. Couples therapy with couples living together has lower risk than couples therapy with couples who live apart. The risk with family therapy will depend on whether all are living in the same house and the degree of cautious behavior of each family member. The degree of out-of-home exposure of each potential patient should be explored and considered, including the extent of exposure of an individual because of their employment and social gathering behavior.

\section{Determining Your Professional Boundaries and Requirements}

According to the National Register/Trust survey data (Sammons et al., 2020b), being vaccinated themselves is viewed as one condition that most psychologists have set as a requirement for resuming in-person therapy. Practicing psychologists, as health service providers, are deemed eligible for vaccination in the earlier stages of the COVID-19 vaccination process in most all states. In the majority of cases, that will mean in the first quarter of 2021. In a smaller number of cases it will mean the second quarter of 2021. Non-practicing psychologists will get vaccinated when eligible under other qualifying conditions (e.g., age, employment setting, health conditions, and so forth). COVID-19 vaccination will probably continue throughout all of 2021.

A psychologist is free to set their own standards for the conditions under which they will see patients face-to-face versus providing care via telepsychology methods. There is no legal requirement that one must see any or all patients seeking 
treatment on an in-person or in-office basis. Most psychologists expect that their future practice will include a mix of inoffice and telepsychology practice, according to the National Register/Trust survey data.

Each psychologist will need to decide whether or not patients will be required to be vaccinated before being eligible to be seen for in-office care in your practice. Patient vaccination in such cases would not be for the protection of the already vaccinated psychologist but rather for the protection of other patients who enter the treatment space after the patient. Among other considerations, allowing unvaccinated patients in treatment areas would increase cleaning and other prevention measures required of a given psychologist in a given setting.

Each psychologist will also need to determine what level of documentation of vaccination they will require. This could be as simple as requiring each patient to sign a document stating that they have received the COVID-19 vaccine, or the psychologist could verify vaccination by viewing the patient's official CDC vaccination card or requiring a photocopy of the official CDC vaccination card. Relevant documentation can be placed in the patient's health care record. Define the procedure for your practice - and then stick to it.

\section{Exploring Vaccination Concerns With Patients}

It is important for the psychologist to create the context in which COVID-19 vaccination, in-office care, and one's practice of psychotherapy are discussed. Doing this can help achieve higher levels of public health and patient vaccination, and less resistance to getting vaccinated. This can be achieved by using four steps or processes: (1) talk with positive expectations, (2) clearly state your practice procedures, (3) use active listening skills to hear the core of any patient doubts or fears, and (4) use motivational interviewing skills to correct misunderstanding and increase motivation for getting vaccinated.

Speak in positive terms, with positive expectations. Assume that the patient has a positive view of the value of vaccinations and is planning to get vaccinated at whatever time they are eligible. Use phrases such as "when you get vaccinated" rather than "if you get vaccinated." Cite the best available evidence, without extending the limits of what is currently known. Reinforce the value of science and empirical health data, research, and findings.

Clearly describe your conditions and expectations for inoffice care. Inform patients of exactly when (or under what exact conditions) you will be providing in-office care. Inform patients of what health status and other health behavior (such as mask usage and vaccinations) you will be expecting as a condition for in-office care in your practice. Inform patients of any vaccination documentation requirements for in-office care. Inform patients of the availability of your telepsychology services for those unable to meet the requirement for in-office care.

Actively listen to doubts and fears. All patients have the right to question health care advice. The essential clinical aspect of this for psychologists is not whether the patient has doubts and fears, but, rather, the core of the concerns and questioning. Will this really help me? How might this hurt me? Is it safe? Identify the concern, and verbalize the affective component involved. Support the patient in their relative degree of uncertainty. The patient has the right to seek the best scientific evidence regarding their specific concern.

Actively explore the patient's understanding of their situation. What is the patient's understanding of the current public health crisis? What is the patient's understanding of the array of options available? Listen to the patient's concerns, and re-state the concern back to the patient to be certain you have heard them correctly. Get their confirmation. If you believe their view is correct, acknowledge your support. If you believe their view is incorrect, invite the patient to further explore their view, state other alternative views, and consider the evidence that supports one or another perspective.

Remember that different information sources have differing standards of so-called "evidence" and differing truth-value or trustworthiness. Introduce such a view to doubting patients who you believe are basing their opinion on questionable sources or rumors.

Express doubt or skepticism about anything you believe is factually incorrect. If the patient responds by telling you that you are wrong or once again tells you their belief, do not argue with them. Rather, drop back and become curious about the patient's beliefs. Invite the patient to join you to further explore the possibilities and the available data.

Some inaccurately held assumptions (such as the FDA being untrustworthy or microchips being in the vaccine) may indicate broader and more general beliefs and related life events to be explored. It is a clinical decision whether to branch into that discussion at the moment or remember it for another time so as to address the more pressing immediate problems.

\section{Four Tips to Remember}

Respect the patient's autonomy. The patient has the right to determine for themselves which health care services they will receive and which they will not. It is important for them to also understand that their decision will come with limitations as well.

Engage in truthful communication. State relevant scientific information, as you know it. Do not overstate what is known. Acknowledge the limits of your knowledge. Commit to engaging in finding additional scientific data as needed. 
Be curious about the patient's views and beliefs. Life experiences shape how we view the world, what we believe, and who we trust. It is always essential in psychotherapy to gain a clear understanding of a patient's lived experiences and the ideas they have developed for coping as a result.

Support an objective, fact-based and scientifically informed way of living. It is always good to reflect on why one believes anything that they believe. Consider who else shares one's beliefs or who (and how many others) hold an alternative belief - and why.

\section{References}

Bloom, D.E., Canning, D. \& Weston, M. (2005). The value of vaccination. World Economics, 6(3), 15-39.

Centers for Disease Control and Prevention. (2020a). CDC COVID Data Tracker. Retrieved December 31, 2020, from https://covid.cdc.gov/ covid-data-tracker/\#cases casesper100klast7days

Centers for Disease Control and Prevention. (2020b). Your Health. November 3, 2020 update. www.cdc.gov/coronavirus/2019-ncov/ your-health/ (accessed January 3, 2021).

Larson, H. J. (2020). Stuck: How Vaccine Rumors Start - and Why They Don't Go Away. New York: Oxford University Press.
Leask, J. (2011). Target the fence-sitters. Nature, 473, 443-445. https:// doi.org/10.1038/473443a

Lewis, J. \& Speers, T. (2003). Misleading media reporting? The MMR story. Nature Reviews, Immunology, 3(11), 913-918.

MacDonald, NE and SAGE Working Group on Vaccine Hesitancy. (2015). Vaccine hesitancy: Definition, scope and determinants. Vaccine, 33(34):4161-4. https://doi.org/10.1016/j.vaccine.2015.04. 036.

Sammons, M. T., VandenBos, G. R. \& Martin, J. N. (2020a) Psychological Practice and the COVID-19 Crisis: A Rapid Response Survey. Journal of Health Service Psychology, 46, 5157. https://doi.org/10.1007/s42843-020-00013-2

Sammons, M.T., VandenBos, G.R., Martin, J.N., Elchert, D. M. (2020b). Psychological Practice at Six Months of COVID-19: A Follow-Up to the First National Survey of Psychologists During the Pandemic. Journal of Health Service Psychology, 46(4). https://doi.org/10. 1007/s42843-020-00024-Z

Publisher's Note Springer Nature remains neutral with regard to jurisdictional claims in published maps and institutional affiliations.

Gary R. VandenBos, $\mathrm{PhD}$, is a clinical psychologist and psychotherapist. $\mathrm{He}$ is the Senior Professional Consultant at the National Register of Health Service Psychologists. He was a Professor II at the University of Bergen (Norway) for 25 years and APA Publisher for $30+$ years. He is the co-author with Bertram Karon of Psychotherapy with Schizophrenics: The Treatment of Choice. 